\title{
Potencial dos resíduos do processamento de camarão para remediação de águas contaminadas com drenagem ácida mineral
}

\section{Potential of shrimp-shell residue in natura for the remediation of mine impacted water (MIW)}

\author{
Dámaris Núñez-Gómez ${ }^{1 *}$, Maria Eliza Nagel-Hassemer, Flávio Rubens Lapolli e \\ Maria Ángeles Lobo-Recio ${ }^{1,2}$ \\ ${ }^{1}$ Departamento de Engenharia Ambiental, Universidade Federal de Santa Catarina - UFSC, \\ Florianópolis, SC, Brasil \\ 2Universidade Federal de Santa Catarina - UFSC, Araranguá, SC, Brasil \\ *damaris_ng@hotmail.com
}

\begin{abstract}
Resumo
A drenagem ácida mineral (DAM) é um dos mais graves impactos ambientais da mineração. Cursos de água impactados pela DAM são avaliados como impróprios acarretando graves impactos ambientais e problemas socioeconômicos severos. Este estudo visa identificar o potencial de um resíduo, casca de camarão, como fonte de quitina e alcalinidade para remediação de águas contaminadas por DAM visando seu posterior reuso para fins secundários não potáveis. Foram efetuados ensaios com a casca de camarão, diferenciando entre corpo (CR) e cabeça (CB), e quitina comercial (QT, $70 \%$ de pureza), para o tratamento de amostras de água superficiais e subterrâneas impactadas da Região Carbonífera Catarinense/SC (Brasil). Realizaram-se ensaios em duplicata e em regime de batelada variando o teor de substrato, tempo de contato e velocidade de agitação. Conseguiu-se a remoção quase total de $\mathrm{Fe}(>92 \%)$ e $\mathrm{Al}(>99 \%)$ e uma remoção de

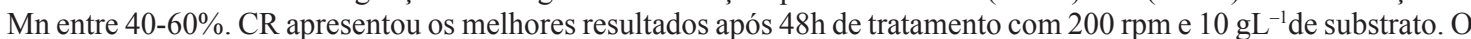
aumento no $\mathrm{pH}$ foi mais significativo nas amostras contendo $\mathrm{CR}$, passando o $\mathrm{pH}$ de $\sim 3$ para $\sim 7$. Os resultados indicaram o potencial da utilização direta da casca de camarão para o tratamento de efluentes para reuso secundário não potável.
\end{abstract}

Palavras-chave: biorremediação, quitina, reuso, sorção, metais dissolvidos.

\begin{abstract}
The mineral acid mine drainage (AMD) is one of the most serious environmental impacts of mining. Watercourses impacted by DAM are assessed as unfit, causing serious environmental consequences and severe socioeconomic problems. The main objective of the present study is to evaluate the potential of a residue, shrimp-shell, as source of chitin and alkalinity for remediation of mine-impacted water (MIW), seeking their subsequent reuse for secondary non-potable reuse. Comparative tests with the shrimp-shells were performed, differentiating between body (CR) and head (CB), and commercial chitin (QT 70\% purity), with samples of surface water and groundwater from the impacted "Região Carbonifera Catarinense" (Brazil). Assays were performed in batch-microcosms with different concentrations of substrate, contact time and stirring speed. Metals were almost removed [Fe (>92\%), $\mathrm{Al}(>99 \%)$, and $\mathrm{Mn}(40-60 \%)]$. CR showed the best results after 48 hours of treatment with $200 \mathrm{rpm}$ and $10 \mathrm{~g} \mathrm{~L}^{-1}$ of substrate. The higher increase in $\mathrm{pH}$ was in the samples with $\mathrm{CR}$, from $\sim 3$ to $\sim 7$. The results indicated the potential of direct use of shrimp shells to treat effluents for secondary non-potable reuse.
\end{abstract}

Keywords: chitin, bioremediation, sorption, reuse, solved metals.

\section{Introdução}

O estudo e descrição dos processos de mineração do carvão tem sido objeto de atenção por parte da comunidade científica e outras instituições devido tanto a sua abrangência quanto à magnitude dos impactos que ocasiona. Dentre os impactos ambientais, o mais grave deles é a poluição dos mananciais hídricos das regiões próximas à de exploração do mineral ${ }^{[1]}$.
A geoquímica das águas ácidas de mina é um fenômeno complexo pela diversidade de processos químicos, físicos e biológicos interferentes ${ }^{[2]}$. Nordstrom e Alpers ${ }^{[3]}$ descreveram o processo de oxidação da pirita $\left(\mathrm{FeS}_{2}\right)$ como o principal responsável pela formação de águas ácidas. Esta oxidação é favorecida nas áreas mineiras pela facilidade com a que $o$ ar entra em contato com os sulfetos presentes nas rochas 
e o incremento da superfície de contato das partículas devido à exposição própria da atividade. A pirita é oxidada e dissociada quando exposta ao ar e à água, liberando $\mathrm{Fe}^{2+}$ em solução (Equação 1). No início é um processo lento que, quando o $\mathrm{pH}$ do meio é ainda relativamente alto, acaba com o ferro liberado precipitado como hidróxido (Equação 2). Progressivamente, a capacidade de neutralização do meio vai diminuindo, deixando de formar hidróxidos e aumentando a atividade do $\mathrm{Fe}^{3+}$, em valores de $\mathrm{pH}$ próximos a 3,5. Neste pH, a oxidação de $\mathrm{Fe}^{2+}$ a $\mathrm{Fe}^{3+}$ é acelerada (Equação 3) em várias ordens de magnitude pela ação bacteriana (Thiobacillus ferrooxidans e Thiobacillus denitrificans) ${ }^{[4]} \mathrm{e}$ ganha importância a oxidação da pirita pelo $\mathrm{Fe}^{3+}$ liberando $\mathrm{Fe}^{2+} \mathrm{e}$ acidez adicional (Equação 4) $)^{[1,3,5-7]}$.

$$
\begin{aligned}
& 2 \mathrm{FeS}_{2}(s)+7 \mathrm{O}_{2}(\mathrm{~g})+6 \mathrm{H}_{2} \mathrm{O}(\mathrm{l}) \leftrightarrow 2 \mathrm{Fe}^{2+}+4 \mathrm{SO}_{4}^{2-}+4 \mathrm{H}_{3} \mathrm{O}^{+} \\
& 4 \mathrm{Fe}^{2+}+18 \mathrm{H}_{2} \mathrm{O}(\mathrm{l})+\mathrm{O}_{2}(\mathrm{~g}) \leftrightarrow 4 \mathrm{Fe}(\mathrm{OH})_{3}(\mathrm{~s})+8 \mathrm{H}_{3} \mathrm{O}^{+} \\
& 4 \mathrm{Fe}^{2+}+\frac{1}{2} \mathrm{O}_{2}(\mathrm{~g})+2 \mathrm{H}_{3} \mathrm{O}^{+} \leftrightarrow 4 \mathrm{Fe}^{3+}+3 \mathrm{H}_{2} \mathrm{O}(\mathrm{l}) \\
& \mathrm{FeS}_{2}(\mathrm{~s})+14 \mathrm{Fe}^{3+}+24 \mathrm{H}_{2} \mathrm{O}(\mathrm{l}) \leftrightarrow 15 \mathrm{Fe}^{2+}+2 \mathrm{SO}_{4}^{2-}+16 \mathrm{H}_{3} \mathrm{O}^{+}
\end{aligned}
$$

Como resultado, os cursos d'água que recebem descargas de drenagem ácida mineral (DAM) apresentam, geralmente, valores de $\mathrm{pH}$ na faixa entre 1,5 e 4,0. A elevada acidez do efluente promove a dissolução de muitos metais e metaloides, tais como $\mathrm{Fe}, \mathrm{Al}, \mathrm{Zn}, \mathrm{Cu}, \mathrm{Cd}, \mathrm{As}$, $\mathrm{Ni}$ e $\mathrm{Pb}$, presentes em outros minerais ${ }^{[3,8,9]}$, muitos dos quais se demonstram tóxicos ou potencialmente tóxicos para os ecossistemas terrestres, aquáticos e, em última instância, para a saúde e qualidade de vida humana. Uma vez impactados os cursos hídricos com DAM é difícil seu controle e remediação, apresentando efeitos em longo prazo e/ou de forma permanente, com impactos tanto no uso das águas superficiais (quali e quantitativamente) quanto das águas subterrâneas, devido à migração dos lixiviados tóxicos. Os sistemas de tratamento convencionais ou tradicionais envolvem neutralização e precipitação dos íons metálicos pela adição de substâncias alcalinas, entretanto com a desvantagem de elevados custos devido à grande produção de lodos tóxicos, às específicas necessidades de manipulação e disposição destes e à elevada quantidade de reagentes necessários para manter o $\mathrm{pH}$ acima de $6,5^{[10]}$.

Frente a este cenário, vem sendo pesquisados e desenvolvidos outros métodos de tratamento. Dentro destes, a biossorção é considerada como técnica promissora para a remoção de íons metálicos. Biossorção é o processo de eliminação de compostos, íons metálicos ou outros materiais utilizando um sorvente inativo (não vivo) de origem biológica, por meio de forças de atração entre o material removido e o biossorvente e envolvendo mecanismos físico-químicos, tais como adsorção física, troca iônica, complexação, co-precipitação e precipitação ${ }^{[11,12]}$. Os biopolímeros são substâncias de ocorrência natural, abundantes, renováveis, não tóxicos e possuem baixo custo.

Dentre os polímeros naturais existentes destaca-se a quitina, disponível em grande quantidade no processamento da indústria pesqueira ${ }^{[13]}$. A quitina é um polissacarídeo nitrogenado, de origem natural, branco, duro e cristalino ${ }^{[14,15]}$. Quimicamente, trata-se de um polímero de alto peso molecular composto por unidades de N-acetil-2-amino-2desoxi-D-glicose unidas entre elas por ligações glicosídicas $\beta(1 \rightarrow 4)$ formando uma cadeia linear com algumas das unidades monoméricas desacetiladas (Figura 1). Encontra-se amplamente distribuída na natureza e, depois da celulose (matéria base do papel), é o segundo polissacarídeo em abundância. Sua principal fonte são os exoesqueletos de muitos crustáceos e moluscos, as asas de insetos artrópodes, as paredes celulares de fungos dentre outras ${ }^{[16]}$.

Muitos autores evidenciaram que a quitina é um excelente adsorvente de íons metálicos, especialmente em condições fortemente ácidas, sendo capaz de remover metais como alumínio, arsênio, cromo, cobre, ferro, manganês, níquel e zinco de soluções aquosas ${ }^{[17-19]}$. Tem sido utilizada com sucesso no tratamento de efluente têxtil ${ }^{[20]}$, no tratamento de água de poç ${ }^{[21,22]}$, no tratamento de efluentes de mineração ${ }^{[2,23,24]}$, em diversas áreas da indústria alimentar, e em formulações farmacêuticas e cosméticas ${ }^{[25,26]}$. O mecanismo da remoção de metais dissolvidos pela quitina tem sido atribuído a processos de adsorção física ou química a valores baixos de $\mathrm{pH}$ e à precipitação de hidróxidos metálicos insolúveis a valores de $\mathrm{pH}$ superiores a $6^{623]}$.

A contínua perda da qualidade socioambiental na Região Carbonífera Catarinense, decorrente do modelo extrativo do carvão adotado historicamente, concentrado no rendimento econômico e sem considerar os custos sociais e ambientais, atinge atualmente proporções alarmantes ${ }^{[27]}$. Avalia-se que dois terços dos recursos hídricos superficiais estão comprometidos, com os rios assoreados e praticamente mortos ${ }^{[28,29]}$. Consequentemente, nas épocas de estiagens acentuam-se os conflitos socioeconômicos pelo direito de acesso a uma

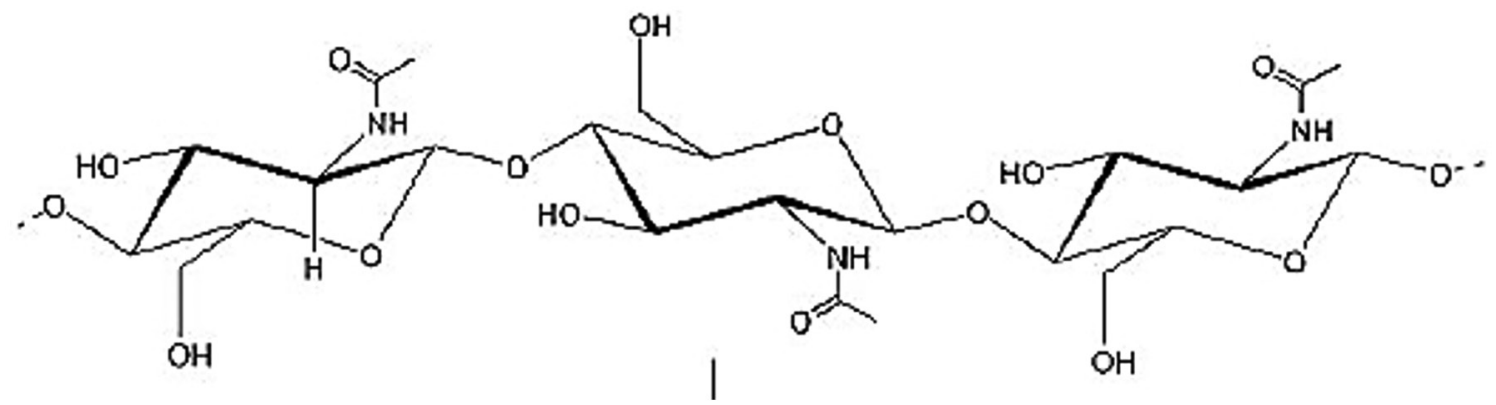

Figura 1. Estrutura do biopolímero quitina. 
água de qualidade necessária para abastecimento humano, porém, também, para múltiplos processos agroindustriais. Convém mencionar que as características observadas em Santa Catarina não são exclusivas desse estado. Sabe-se que a mineração é uma das atividades humanas que mais contribuem para a alteração da superfície terrestre, provocando expressivos impactos sobre a água, o ar, o solo, o subsolo e a paisagem como um todo ${ }^{[27]}$.

Por outro lado, a carcinicultura catarinense apresentou um rápido incremento com a introdução do Litopenaeus vannamei, passando de 50 toneladas em 1998, para 3.251 no ano de 2003, o que representou $3,6 \%$ da carcinicultura total brasileira e um crescimento de $97 \%$ face o ano anterior (produção de 1.650 toneladas) ${ }^{[30]}$. Entre 2004 e 2005, os viveiros catarinenses foram acometidos pelo vírus da mancha branca (White Spot Sindrome Virus - WSSV) o que afetou significativamente a produção, forçando em muitos casos o abandono da prática e cesse da atividade na região. Atualmente, o Estado de Santa Catarina pretende-se perfilar como um grande produtor de camarão através do uso e fomento de políticas públicas, legislação ambiental específica e monitoramentos periódicos.

O objetivo deste estudo foi avaliar o potencial deste resíduo, casca de camarão, como fonte de quitina e alcalinidade para remediação de águas contaminadas com DAM visando seu posterior reuso, em conformidade com os padrões legais pertinentes, como água para fins secundários não potáveis agropecuários, industriais e urbanos, preservando assim os mananciais de água de qualidade para usos potáveis de abastecimento populacional.

\section{Materiais e Métodos}

\subsection{Substratos bioadsorventes (Quitina)}

Para os ensaios comparativos de substrato utilizou-se quitina comercial (QT) com 70\% de grau de pureza da marca Polymar e casca de camarão, diferenciando entre corpo (CR) e cabeça (CB) que apresentam diferentes concentrações de $\mathrm{CaCO}_{3}$ e quantidade de matéria orgânica associada ${ }^{[31,32]}$. Os substratos CR e CB foram lavados com água corrente de forma meticulosa, para eliminar restos de matéria orgânica e outros materiais grosseiros, e secados em estufa por 72 horas, a $100{ }^{\circ} \mathrm{C}$ nas primeiras 48 horas, e a $50^{\circ} \mathrm{C}$ nas últimas $24 \mathrm{~h}$. Após esse processo, foram pulverizados em liquidificador e peneirados para promover maior homogeneidade $\mathrm{e}$ superfície de contato. Todos os substratos foram mantidos

Tabela 1. Coordenadas de localização dos pontos amostrais das águas usadas nos ensaio.

\begin{tabular}{lcc}
\hline & MLD (Siderópolis/ & RS (Criciúma/SC) \\
& SC) & \\
\hline \multirow{2}{*}{ Localização (GPS) } & $28.35 .678 \mathrm{~S}$ & $28.41 .023 \mathrm{~S}$ \\
& $49.27 .641 \mathrm{~W}$ & $49.24 .893 \mathrm{~W}$ \\
\hline
\end{tabular}

em dessecador de vidro à vácuo durante o processo para evitar a absorção de umidade.

\subsection{Pontos amostrais da água impactada}

As amostras líquidas para realização dos ensaios comparativos foram obtidas de dois locais diferentes da Bacia Hidrográfica do Rio Araranguá (Região Hidrográfica 10: "Extremo Sul Catarinense"), situada dentro da região Carbonífera Catarinense no Sul do Estado de Santa Catarina, sendo estas: Manancial Língua do Dragão (MLD) e Rio Sangão (RS) (Tabela 1). O primeiro deles corresponde a um manancial subterrâneo impactado antes da sua emanação superficial pela grande quantidade de pirita exposta na área devido à inversão do perfil edáfico, decorrente de antigas atividades de extração de carvão mineral a céu aberto. O Rio Sangão, pelo contrário, trata-se de um curso hídrico superficial impactado com os efluentes ácidos da mineração dentre outros efluentes industriais e urbanos. As amostras foram coletadas em pontos de fácil acesso, em garrafas de polipropileno de $5 \mathrm{~L}$ de capacidade (não estéreis), transportadas e mantidas a temperatura constante de $4{ }^{\circ} \mathrm{C}$. As amostras foram caracterizadas no mesmo dia da coleta em termos de $\mathrm{pH}$ ( $\mathrm{pHmetro}$ ThermoFisher, Scientiphic Orion 3Stars), ânions (Cromatografia iônica, Dionex ICS-1000) e espécies metálicas e metalóides (ICP-MS, Perkin Elmer, Nexlon 300D).

\subsection{Experimentos de remediação}

Foram efetuados diferentes ensaios em regime de batelada visando avaliar a influência na remediação das águas do $\mathrm{pH}$, da relação quantidade de substrato/volume de água, do tempo de contato e da velocidade de agitação.

Todos os ensaios foram efetuados em duplicata e em banho termostático (Dubnoff 252), usando erlenmeyers de polipropileno de $250 \mathrm{~mL}$ de capacidade total, não estéreis, tampados com papel film para evitar entrada de impurezas ambientais e/ou água do banho termostático. Após o período de incubação de cada um dos ensaios, as amostras foram filtradas com membranas de acetato de celulose de porosidade $0,45 \mu \mathrm{m}$ e, posteriormente, foi medido o $\mathrm{pH}$ final e as concentrações residuais totais de metais para cada uma das soluções. Estas concentrações foram determinadas mediante espectroscopia UV-VIS $(\mathrm{HACH}$, DR/4000U). Os reagentes utilizados para a quantificação das espécies analisadas foram provenientes de kits para espectrofotometria visível da marca $\mathrm{HACH}^{\circledR}$. As espécies analisadas no espectrofotômetro e as faixas de detecção de cada método utilizado estão apresentadas na Tabela 2.

$\mathrm{O}$ efeito da quantidade e grau de pureza dos substratos (teor de $\mathrm{CaCO}_{3}$ ) no $\mathrm{pH}$ foi determinado mediante o contato de $50 \mathrm{~mL}$ de água dos locais amostrais (MLD e RS) com diferentes teores dos substratos CB, CR e QT ( 5 e $10 \mathrm{~g} \mathrm{~L}^{-1}$ ). Estas quantidades foram selecionadas com base na literatura $\mathrm{e}$

Tabela 2. Elementos analisados por colorimetria.

\begin{tabular}{cccc}
\hline Elemento & Método de detecção & Espécie medida & Faixa detecção \\
\hline Alumínio & Aluminom Method & $\mathrm{Al}^{3+}$ & $0 \mathrm{a} 0,8 \mathrm{mg} \mathrm{L}^{-1}$ \\
Ferro & 1,10 Phenanthroline Method & Fe total & 0 a $3,00 \mathrm{mg} \mathrm{L}^{-1}$ \\
Manganês & Periodate Oxidation Method & Mn total & $0,001 \mathrm{mg} \mathrm{L}^{-1}$ \\
\hline
\end{tabular}


em função das altas concentrações de algumas espécies como o Ferro ${ }^{[2,19,23,33-35]}$. Para o controle de qualidade, os ensaios foram realizados em duplicata e com brancos (amostras líquidas sem substratos). As soluções foram tratadas por 24 horas a temperatura ambiente $\left(22 \pm 2{ }^{\circ} \mathrm{C}\right)$ sem agitação.

O efeito do tempo de contato substrato/água e da agitação das amostras determinou-se pelo contato de 1,5 $\mathrm{g}$ de substrato (CB, CR e QT) com $150 \mathrm{~mL}$ das amostras $\left(10 \mathrm{~g} \mathrm{~L}^{-1}\right)$ em erlenmeyers de polipropileno, sob agitação (100, 150 e $200 \mathrm{rpm})$ e temperatura controlada $\left(22 \pm 1^{\circ} \mathrm{C}\right)$. Amostras para cada substrato foram filtradas após 24 e 48 horas de experimento. Um frasco sem substrato serviu como controle em cada uma das condições testadas. Esta metodologia foi baseada na literatura ${ }^{[19]}$ com algumas modificações.

\section{Resultados e Discussão}

\subsection{Caracterização inicial das amostras}

Os dados analíticos das amostras (Tabela 3) permitem visualizar que, apesar do alto grau de contaminação de ambas por DAM, a água de origem subterrânea (MLD) está mais poluída que a água de origem superficial (RS).

Com base na caracterização inicial das amostras, determinou-se que Al, Fe e Mn seriam as espécies catiônicas monitoradas nos ensaios de remoção com quitina, por se encontrarem muito acima dos níveis de risco conforme estabelecido na legislação (Tabela 3). Os ânions foram também monitorados, especialmente o sulfato, dado seu elevadíssimo teor nas águas objeto de estudo.

\subsection{Efeito da quantidade dos diferentes substratos no pH das amostras}

Os resultados obtidos na elevação do $\mathrm{pH}$ das amostras após contato com diferentes quantidades dos substratos durante 24 horas encontram-se na Tabela 4.

Os incrementos de $\mathrm{pH}$ observados nas amostras encontram-se conformes com a literatura ${ }^{[2,19,23,33-35]}$. Os resultados mostram que nos casos estudados a elevação do $\mathrm{pH}$ das amostras foi maior com casca de camarão que com quitina comercial. O experimento demonstrou, portanto, que a casca de camarão (CB e CR) é mais efetiva para a remoção da acidez das amostras que a quitina comercial.

Com base nos resultados, determinou-se a utilização de 10 g substrato $\mathrm{L}^{-1}$ de amostra para os subsequentes ensaios por fornecer maior elevação do $\mathrm{pH}$, considerando a potencial sinergia dos processos de remoção de Al, Mn e Fe via adsorção em quitina e via precipitação como hidróxidos, assim como a eficiência e aprimoramento do tratamento de águas impactadas por DAM.

\subsection{Efeito do tempo de contato substrato/água na remoção de acidez, metais e sulfato}

No segundo experimento, as amostras de água foram postas em contato com os substratos $\left(10 \mathrm{~g} \mathrm{~L}^{-1}\right)$ e agitadas a $100 \mathrm{rpm}$ durante 24 e 48 horas, no intuito de determinar a influência do tempo de contato no processo de remoção de espécies metálicas e neutralização da acidez das águas. Os resultados estão representados na Tabela 5. Como esperado, em todos os casos o $\mathrm{pH}$ das amostras aumentou após os tratamentos, sendo que após $48 \mathrm{~h}$ apresentou valores ligeiramente superiores com respeito aos valores após $24 \mathrm{~h}$ de

Tabela 3. Caracterização inicial das amostras e comparação com os valores legais máximos permitidos.

\begin{tabular}{|c|c|c|c|c|}
\hline & MLD (Siderópolis/SC) & RS (Criciúma/SC) & $\begin{array}{c}\text { Resolução CONAMA } \\
430 / 2011^{\mathrm{a}}\end{array}$ & $\begin{array}{c}\text { Resolução CONAMA } \\
\text { 357/2005 }^{\text {b }}\end{array}$ \\
\hline pH & 2,82 & 3,04 & $5-9$ & $6-9$ \\
\hline Arsênico (mg L L $\left.{ }^{-1}\right)$ & 0,00273 & 0,00246 & 0,5 & 0,033 \\
\hline Alumínio (mg L $\left.{ }^{-1}\right)$ & 75,6 & 35,92 & $\mathrm{NC}^{\mathrm{c}}$ & 0,2 \\
\hline Cádmio (mg L $\left.{ }^{-1}\right)$ & 0,0015 & 0,00144 & 0,2 & 0,01 \\
\hline Chumbo (mg L L $\left.{ }^{-1}\right)$ & 0,00048 & 0,00282 & 0,5 & 0,033 \\
\hline Cobalto $\left(\mathrm{mg} \mathrm{L}^{-1}\right)$ & 0,34536 & 0,07844 & $\mathrm{NC}^{\mathrm{c}}$ & 0,2 \\
\hline Cobre $\left(m g \mathbf{L}^{-1}\right)$ & 0,00009 & 0,01372 & 1 & 0,05 \\
\hline Ferro $\left(\mathrm{mg} \mathrm{L}^{-1}\right)$ & 514,58 & 56,3 & 15 & 5 \\
\hline Manganês (mg L $\mathbf{L}^{-1}$ ) & 29,36 & 2,72 & 1 & 0,5 \\
\hline Níquel (mg L L $\left.{ }^{-1}\right)$ & 0,4372 & 0,11715 & 2 & 0,025 \\
\hline $\operatorname{Acetato}\left(\mathrm{mg} \mathrm{L}^{-1}\right)$ & 2,021 & 2,258 & $\mathrm{NC}^{\mathrm{c}}$ & $\mathrm{NC}^{\mathrm{c}}$ \\
\hline Cloreto $\left(\mathrm{mg} \mathrm{L}^{-1}\right)$ & 13,862 & 13,918 & $\mathrm{NC}^{\mathrm{c}}$ & 250 \\
\hline Fosfato $\left(\mathrm{mg} \mathrm{L}^{-1}\right)$ & 1,352 & 0,281 & $\mathrm{NC}^{\mathrm{c}}$ & $\mathrm{NC}^{\mathrm{c}}$ \\
\hline Nitrato $\left(\mathrm{mg} \mathrm{L}^{-1}\right)$ & 0,287 & 1,448 & $\mathrm{NC}^{\mathrm{c}}$ & 10 \\
\hline Sulfato (mg L $\left.\mathbf{L}^{-1}\right)$ & 2685,925 & 617,26 & $\mathrm{NC}^{\mathrm{c}}$ & 250 \\
\hline
\end{tabular}

${ }^{\mathrm{a}}$ Dispõe sobre as condições e padrões de lançamento de efluentes. ${ }^{\mathrm{b}}$ Dispõe sobre a classificação dos corpos de água e diretrizes ambientais para o seu enquadramento (Classe 3 e 4 ). ${ }^{\mathrm{c}} \mathrm{NC}=$ Não Considerado.

Tabela 4. Variação do pH das amostras após tratamento com os substratos.

\begin{tabular}{|c|c|c|c|c|c|c|c|}
\hline \multirow{2}{*}{ Amostra } & \multirow{2}{*}{ pH Inicial } & \multicolumn{3}{|c|}{$5 \mathrm{~g} \mathrm{~L}^{-1}$} & \multicolumn{3}{|c|}{$10 \mathrm{~g} \mathrm{~L}^{-1}$} \\
\hline & & CB & CR & QT & CB & CR & QT \\
\hline MLD & 2,88 & 4,63 & 4,46 & 3,45 & 6,83 & 6,63 & 4,75 \\
\hline RS & 3,04 & 7,12 & 7,21 & 5,35 & 7,81 & 7,93 & 6,19 \\
\hline
\end{tabular}


agitação, mantendo-se na mesma faixa que no experimento anterior. As amostras tratadas com CR apresentaram maior incremento em $48 \mathrm{~h}$, se estabilizando em valores próximos à neutralidade (Tabela 5).

Na remoção de espécies metálicas, as águas dos pontos amostrais apresentaram comportamentos diferentes para os substratos em função da sua composição inicial e características próprias (teor de espécies metálicas, presença de matéria orgânica, material suspenso, etc.). Para MLD (Tabela 5), após $24 \mathrm{~h}$ obteve-se uma remoção de $\mathrm{Fe}$ e $\mathrm{Al}$ em torno de $80 \%$ e de cerca de $30 \%$ para o Mn. Estes porcentuais de remoção melhoraram significativamente após $48 \mathrm{~h}$ de tratamento, atingindo valores $>92 \%$ para $\mathrm{Fe}, 99 \%$ para $\mathrm{Al}$ e $52 \%$ para $\mathrm{Mn}$. Cabe destacar que em $48 \mathrm{~h}$ e com CR, a concentração final de $\mathrm{Fe}\left(14,58 \mathrm{mg} \mathrm{L}^{-1}\right)$ atingiu os padrões legais estabelecidos na Resolução CONAMA 430/2011 para emissão de efluentes $\left(15 \mathrm{mg} \mathrm{L}^{-1}\right)^{[36]}$ e que a concentração residual do $\mathrm{Al}\left(0,1 \mathrm{mg} \mathrm{L}^{-1}\right)$ permite caracterizar o efluente com respeito a este parâmetro como de Classe 3 segundo a Resolução CONAMA 357/2005 (0,2 mg L $\left.)^{-1}\right)^{[37]}$.

Já para as amostras do RS, a média dos porcentuais de remoção após $24 \mathrm{~h}$ foi de $95 \%$ para $\mathrm{Fe}, 96 \%$ para $\mathrm{Al}$ e $71 \%$ para $\mathrm{Mn}$, aumentando para $97 \% \mathrm{Fe}, 99 \% \mathrm{Al}$ e $80 \%$ Mn após $48 \mathrm{~h}$ (Tabela 5). Neste caso, após $48 \mathrm{~h}$ com os três substratos atingiram-se os níveis estabelecidos na legislação para $\mathrm{Fe}, \mathrm{Al}$ e Mn, tanto de emissão de efluentes quanto para classificação como classe 3. A QT mostrou as maiores taxas de remoção para $\mathrm{Al}$, e Fe, sendo que para $\mathrm{Mn}$ foi a $\mathrm{CB}$, seguida pelo CR. Estes melhores resultados de remoção são atribuídos ao menor grau de contaminação das águas do Rio Sangão com respeito às águas do manancial da Língua do Dragão.

Em contraste, a concentração de sulfato não apresentou modificações após os tratamentos $\left(2.685,92 \mathrm{mg} \mathrm{L}^{-1}\right.$ para MLD e $617,26 \mathrm{mg} \mathrm{L}^{-1}$ para RS), indicando que a quitina, nas condições testadas, não é um agente adequado para a remoção deste contaminante.

Por outro lado, após $48 \mathrm{~h}$ de contato com as águas, a CB transformou-se em todos os casos em um substrato gelatinoso, possivelmente pela presença de matéria orgânica, que limitou a remoção de metais e neutralização da acidez das amostras, além de dificultar o processo de filtração.

Com base nos resultados, determinou-se $48 \mathrm{~h}$ como o tempo apropriado para o tratamento e a utilização como substratos de CR e QT para os subsequentes ensaios, descartando a CB pela modificação na sua natureza sólida inicial, que poderia interferir no adequado funcionamento de sistemas que operam em contínuo.

\subsection{Efeito da agitação das amostras na remoção de acidez e metais}

Com objetivo de determinar a influência da agitação no processo de remoção, novos ensaios foram efetuados com CR e QT, mantendo as amostras com diferentes agitações (100, 150 e $200 \mathrm{rpm})$ durante 48h. Estes experimentos indicaram a sinergia positiva da agitação mecânica contínua no tratamento global em termos de eficácia de remoção de espécies metálicas. Para as amostras MLD e RS obtiveram-se com $200 \mathrm{rpm}$ as melhores taxas de remoção de $\mathrm{Fe}$ (>97\%) e $\mathrm{Al}(>99 \%$ ) (Tabela 6 ) quando comparadas com os ensaios a 100 e $150 \mathrm{rpm}$. Os resultados se enquadram em ambos os casos dentro dos padrões legais pertinentes, destacando a remoção total do Al em RS após 48h de tratamento com QT.

Por sua vez, o Mn, para os pontos amostrais, mostrou uma tendência variável e disposição ao aumento quando tratado com QT a $200 \mathrm{rpm}$, resultando em concentrações finais superiores as iniciais (Tabela 6). Este fato poderia ser devido à existência do Mn como constituinte dos próprios substratos, o que acarretaria sua posterior liberação ao meio durante o tratamento com agitação vigorosa, dado que, de acordo com a bibliografia, a quitina apresenta $\mathrm{Mn}$, em diferentes teores, na sua composição inicial ${ }^{[23,38]}$. Assim, uma pesquisa similar testou a adsorção de Mn em substratos derivados de quitina em quantidade de $5 \mathrm{~g} \mathrm{~L}^{-1}$, não observando remoção do $\mathrm{Mn}$ para $\mathrm{pH}>5$, porém quantificando a capacidade máxima de adsorção do polímero em $0,165 \mathrm{mg} \mathrm{g}^{-1}$ a pH 5,4 e $0,981 \mathrm{mg} \mathrm{g}^{-1}$ a pH 8,7 $7^{[23]}$. De acordo com isto, é possível

Tabela 5. pH e concentração final em águas do MLD (Manancial Língua do Dragão) e RS (Rio Sangão) após 24 e 48 horas de tratamento com $10 \mathrm{~g} \mathrm{~L}^{-1}$ dos substratos CB (casca da cabeça de camarão), CR (casca do corpo de camarão) e QT (quitina comercial).

\begin{tabular}{|c|c|c|c|c|c|c|c|c|c|c|c|c|}
\hline \multirow{3}{*}{$\begin{array}{c}\text { Amostra } \\
\text { Tempo contato } \\
\text { Substrato }\end{array}$} & \multicolumn{6}{|c|}{ MLD } & \multicolumn{6}{|c|}{ RS } \\
\hline & \multicolumn{3}{|c|}{24 horas } & \multicolumn{3}{|c|}{48 horas } & \multicolumn{3}{|c|}{24 horas } & \multicolumn{3}{|c|}{48 horas } \\
\hline & CB & CR & QT & CB & CR & QT & CB & CR & QT & CB & CR & QT \\
\hline $\mathrm{Fe}\left(\mathrm{mgL}^{-1}\right)$ & 72,92 & 80,42 & 56,83 & 39,2 & 14,58 & 65,66 & 6,4 & 0,71 & 0,36 & 1,58 & 2,8 & 0,14 \\
\hline $\mathrm{Al}\left(\mathrm{mgL}^{-1}\right)$ & 7 & 30,5 & 9,5 & 0,29 & 0,1 & 0,55 & 0,5 & 1,5 & 1,5 & 0,06 & 0,08 & 0,01 \\
\hline $\operatorname{Mn}\left(\mathrm{mgL}^{-1}\right)$ & 18 & 22,4 & 38,8 & 11 & 4,6 & 26 & 0,59 & 0,64 & 1,05 & 0,26 & 0,37 & 0,95 \\
\hline $\mathrm{pH}$ & 5,2 & 6,36 & 4,65 & 5,82 & 6,43 & 4,99 & 7,71 & 7,58 & 6,67 & 7,82 & 7,31 & 6,72 \\
\hline
\end{tabular}

Tabela 6. pH e concentração final de metais em águas do MLD (Manancial Língua do Dragão) e RS (Rio Sangão) após 48 horas de tratamento com $10 \mathrm{~g} \mathrm{~L}^{-1}$ dos substratos CB (casca da cabeça de camarão), CR (casca do corpo de camarão) e QT (quitina comercial) a diferentes agitações.

\begin{tabular}{|c|c|c|c|c|c|c|c|c|c|c|c|c|}
\hline \multirow{3}{*}{$\begin{array}{c}\text { Amostra } \\
\text { Agitação (rpm) } \\
\text { Substrato }\end{array}$} & \multicolumn{6}{|c|}{ MLD } & \multicolumn{6}{|c|}{$\mathrm{RS}$} \\
\hline & \multicolumn{2}{|c|}{100} & \multicolumn{2}{|c|}{150} & \multicolumn{2}{|c|}{200} & \multicolumn{2}{|c|}{100} & \multicolumn{2}{|c|}{150} & \multicolumn{2}{|c|}{200} \\
\hline & CR & QT & CR & QT & CR & QT & CR & QT & CR & QT & CR & QT \\
\hline $\mathrm{Fe}\left(\mathrm{mgL}^{-1}\right)$ & 14,58 & 65,66 & 7 & 2,78 & 0,8 & 0,87 & 2,8 & 0,14 & 0,37 & 3,09 & 0,78 & 0,35 \\
\hline $\mathrm{Al}\left(\mathrm{mgL}^{-1}\right)$ & 0,1 & 0,55 & 0,16 & 1,3 & 0,02 & 0,51 & 0,08 & 0,01 & 0,31 & 0,1 & 0,03 & 0 \\
\hline $\operatorname{Mn}\left(\mathrm{mgL}^{-1}\right)$ & 4,6 & 26 & 1 & 14,8 & 7,4 & 82 & 0,34 & 1,05 & 0,37 & 0,76 & 0,2 & 4,01 \\
\hline $\mathrm{pH}$ & 6,43 & 4,99 & 6,1 & 5,13 & 7,27 & 4,98 & 7,31 & 6,72 & 7,37 & 4,67 & 7,6 & 6,14 \\
\hline
\end{tabular}


que o CR tenha adsorvido Mn da amostra RS por apresentar o pH mais elevado, 7,5 (Tabela 6). No caso da água MLD, uma agitação de $150 \mathrm{rpm}$ promoveu uma maior remoção de manganês, embora as remoções de $\mathrm{Al}$ e Fe foram mais baixas que com $200 \mathrm{rpm}$.

Em relação ao $\mathrm{pH}$, os resultados confirmaram um maior incremento nas amostras tratadas com casca de camarão (CR) que nas tratadas com quitina comercial (QT) decorrente, além da remoção do alumínio (ácido de Lewis), do $\mathrm{CaCO}_{3}$ constituinte da casca de camarão, o qual contribuiu para elevar a alcalinidade das amostras, em maior grau quanto maior a agitação. $\mathrm{O}$ maior $\mathrm{pH}$ final nos ensaios foi de 7,27 nas amostras com pH inicial de 2,72 (MLD), e de 7,6 em amostras com pH inicial de 3,04 (RS). Nos controles (amostras sem substrato) o $\mathrm{pH}$ final foi muito próximo ao $\mathrm{pH}$ inicial.

\section{Conclusão}

A casca de camarão CR mostrou-se efetiva para a neutralização da elevada acidez das águas superficiais e subterrâneas impactadas com drenagem ácida mineral, em níveis superiores à quitina comercial (QT), devido ao maior conteúdo de $\mathrm{CaCO}_{3}$ da primeira. Remoções quase totais de $\mathrm{Al}$ e $\mathrm{Fe}$ foram atingidas para ambos os substratos e foi obtida uma remoção considerável de Mn no caso do uso de CR. Em geral, as melhores condições de tratamento encontradas foram $10 \mathrm{~g} \mathrm{CR} \mathrm{L}^{-1}$ água, $48 \mathrm{~h}$ de tempo de contato e velocidade de agitação de $200 \mathrm{rpm}$.

Os experimentos confirmaram a influência do $\mathrm{pH}$ no processo, sugerindo que em $\mathrm{pH}$ baixo a remoção acontece via adsorção e em $\mathrm{pH}$ perto da neutralidade via adsorção e/ou precipitação de hidróxidos. Os resultados indicaram o potencial da utilização direta da casca de camarão para a obtenção de efluentes com características adequadas para seu reuso em usos não potáveis. Partindo destes dados preliminares, estão sendo efetuadas análises estatísticas, via planejamento fatorial, para determinar as condições ideais do tratamento. É de destacar a viabilidade econômica e o caráter sustentável do processo, já que é usado como agente de tratamento um resíduo sem valor comercial cujo descarte acarreta sérios problemas à indústria de processamento de camarão. Mais estudos devem ser realizados visando conhecer as cinéticas de remoção dos metais e no intuito de conseguir uma ótima remoção de Mn e diminuição dos teores de sulfato presentes nas amostras, o qual possibilitaria o enquadramento legal das águas e viabilizaria sua reutilização pela população.

\section{Referências}

1. Ortiz, L., \& Teixeira, E. C. (2002). Meio ambiente e carvão: impactos da exploração e utilização. Porto Alegre: FEPAM.

2. Daubert, L. N., \& Brennan, R. A. (2007). Passive remediation of acid mine drainage using crab shell chitin. Environmental Engineering Science, 24(10), 1475-1480. http://dx.doi. org/10.1089/ees.2006.0199.

3. Nordstrom, D., \& Alpers, C. (1999). Geochemistry of acid mine waters. In G. Plumlee, \& M. J. Logsdon (Ed.), The environmental geochemistry of mineral deposists (Reviews in Economic Geology, Vol. 6A, pp. 133-156). Littleton: Society of Economic Geologists.
4. Rubio, J. (1998). Tratamento de minério. Rio de Janeiro: CETEM.

5. Skousen, J., Rose, A., Geidel, G., Foreman, J., Evans, R., \& Hellier, W. (1998). Handbook of technologies for avoidance and remediation of acid mine drainage. Morgantown: National Mine Land Reclamation Center.

6. Brady, K. B. C., Hawkins, J. W., Miller, K., \& Cuddeback, J. E. (2001). EPA-821-B-01-010: coal remining: best management practices guidance manual. Washington, DC: Environmental Protection Agency.

7. López Pamo, E. (2002). Tratamientos pasivos de drenajes ácidos de mina: estado actual y perspectivas de futuro. Boletín Geológico y Minero, (113), 3-21.

8. Morin, K. A., \& Hutt, N. M. (1997). Environmental geochemistry of minesite drainage: practical theory and case studies. Canada: MDAG Publishing.

9. Mills, C. (1995). Acid base accounting (ABA), metal leaching tests procedures and kinetic testwork interpretation. Vancouver: Acid Rock Drainage of Mining Association of British Columbia. Recuperado em 22 de abril de 2014, de http://technology. infomine.com/enviromine/ard/Acid-Base\%20Accounting/ ABAdiscussion.htm\#Mills.

10. Velazquez, C. L. (2006). Quitina y quitosano: materials del pasado para el presente y el futuro. Avances en Química, 1(2), 15-21. Recuperado em 22 de abril de 2014, de http://www. saber.ula.ve/bitstream/123456789/18309/2/divulg-1-2-2006. pdf.

11. Farooq, U., Kozinski, J., Khan, M., \& Athar, M. (2010). Biosorption of heavy metal ions using wheat based biosorbents: a review of the recent literature. Bioresource Technology, 101(14), 5043-5053. http://dx.doi.org/10.1016/j.biortech.2010.02.030.

12. Veglio, F., \& Beolchini, F. (1997). Removal of metals by bisorption: a review. Hydrometallurgy, 44(3), 301-316. http:// dx.doi.org/10.1016/S0304-386X(96)00059-X.

13. Spinelli, V. A. (2001). Quitosana, polieletrólito natural para o tratamento de água potável (Disertação de mestrado). Universidade Federal de Santa Catarina, Florianópolis.

14. Braconnot, H. (1811). Sur la nature des champignons. Annals of Chemistry and Physics, 79, 265-304.

15. Odier, A. (1823). Mémoire sur la composition chimique des paties cornées des insectes. Mémoires de la Société d'Histoire Naturelle, 1, 29-42. Recuperado em 22 de abril de 2014, de http://www.biodiversitylibrary.org/item/23445\#page/45/ mode/1up.

16. Dutta, P. K., Ravikumar, M. N. V., \& Dutta, J. (2002). Chitin and chitosan for versatile applications. Journal of Macromolecular Science, Part C: Polymer Reviews, 42(3), 307-354. http:// dx.doi.org/10.1081/MC-120006451.

17. Mcafee, B. J., Gould, W. D., Nadeau, J. C., \& da Costa, A. C. (2001). Biosorption of metal ions using chitosan, chitin, and biomass of Rhizopus oryzae. Separation Science and Technology, 36(14), 3207-3222. http://dx.doi.org/10.1081/ SS-100107768.

18. Franco, L. D. O., Maia, R. D. C. C., Porto, A. L. F., Messias, A. S., Fukushima, K., \& Campos-Takaki, G. M. D. (2004). Heavy metal biosorption by chitin and chitosan isolated from Cunninghamella elegans (IFM 46109). Brazilian Journal of Microbiology, 35(3), 243-247. http://dx.doi.org/10.1590/ S1517-83822004000200013.

19. Vijayaraghavan, K., Jegan, J. R., Palanivelu, K., \& Velan, M. (2005). Nickel recovery from aqueous solution using crab shell particles. Adsorption Science and Technology, 23(4), 303-312. http://dx.doi.org/10.1260/0263617054770002.

20. Simionato, J. I., Paulino, A. T., Garcia, J. C., \& Nozaki, J. (2006). Adsorption of aluminium from wastewater by chitin and chitosan produced from silkworm chrysalides. Polymer 
International, 55(11), 1243-1248. http://dx.doi.org/10.1002/ pi.2070.

21. Lobo-Recio, M. Á., Lapolli, F. R., Belli, T. J., Folzke, C. T., \& Tarpani, R. R. Z. (2013). Study of the removal of residual aluminum through the biopolymers carboxymethylcellulose, chitin, and chitosan. Desalination and Water Treatment, 51 (79), 1735-1743. http://dx.doi.org/10.1080/19443994.2012.715 133.

22. Tarpani, R. R. Z. (2012). Remoção de alumínio monomérico de água para abastecimento através da ação da carboximetilcelulose e da quitina (Dissertação de mestrado). Universidade Federal de Santa Catarina, Florianópolis.

23. Robinson-Lora, M. A., \& Brennan, R. A. (2009). Efficient metal removal and neutralization of acid mine drainage by crab-shell chitin under batch and continuous-flow conditions. Bioresource Technology, 100(21), 5063-5071. http://dx.doi. org/10.1016/j.biortech.2008.11.063. PMid:19560340.

24. Gamage, A., \& Shahidi, F. (2007). Use of chitosan for the removal of metal ion contaminants and proteins from water. Food Chemistry, 104(3), 989-996. http://dx.doi.org/10.1016/j. foodchem.2007.01.004.

25. Peter, M. G. (1995). Applications and environmental aspects of chitin and chitosan. Journal of Macromolecular Science, Part A: Pure and Applied Chemistry, 32(4), 629-640. http:// dx.doi.org/10.1080/10601329508010276.

26. Gildberg, A., \& Stenberg, E. (2001). A new process for advanced utilisation of shrimp waste. Process Biochemistry, 36(8), 809812. http://dx.doi.org/10.1016/S0032-9592(00)00278-8.

27. Possamai, F. P., Viana, E., Schulz, H. E., Costa, M. M. D., \& Casagrande, E. (2007). Lixões inativos na região carbonífera de Santa Catarina: análise dos riscos à saúde pública e ao meio ambiente. Ciencia \& Saude Coletiva, 12(1), 171-179. http://dx.doi.org/10.1590/S1413-81232007000100020. PMid:17680068.

28. Estudos Ambientais Ltda. (1995). Influência da poluição da mineração de carvão no cultivo de camarão da empresa lusomar Araranguá - Santa Catarina. Porto Alegre: Estudos Ambientais Ltda.

29. Muñoz Espinosa, H. R. (2001). Impactos e conflitos na gestão de recursos hídricos do sul de Santa Catarina, Brasil. In UNESCO, Agua, vida y desarrollo (pp. 1-12). Santiago: Instituto Interamericano de Cooperación para la Agricultura.
30. Revista da Associação Brasileira da Carcinicultura-ABCC. (2014). Recuperado em 22 de abril de 2014, de http://issuu.com/ marineumarocha/docs/revista_edi_o_janeiro_de_2014.

31. Moura, C. M. D., Muszinski, P., Schmidt, C., Almeida, J., \& Pinto, L.A. A. (2006). Quitina e quitosana produzidas a partir de resíduos de camarão e siri: avaliação do processo em escala piloto. Vetor, 16(1/2), 37-45. Recuperado em 22 de abril de 2014, de http://www.researchgate.net/publication/270049228.

32. Cardoso, M. (2008). Contribuição ao estudo da reação de desacetilação de quitina: estudos da desacetilação assistida por ultra-som de alta potência (Tese de doutorado). Universidade de São Paulo, São Carlos.

33. Hawke, D. J., Sotolongo, S., \& Millero, F. J. (1991). Uptake of $\mathrm{Fe}$ (II) and Mn (II) on chitin as a model organic phase. Marine Chemistry, 33(3), 201-212. http://dx.doi.org/10.1016/03044203(91)90067-7.

34. Benguella, B., \& Benaissa, H. (2002). Cadmium removal from aqueous solutions by chitin: kinetic and equilibrium studies. Water Research, 36(10), 2463-2474. http://dx.doi.org/10.1016/ S0043-1354(01)00459-6. PMid:12153012.

35. Zhou, D., Zhang, L., Zhou, J., \& Guo, S. (2004). Cellulose/ chitin beads for adsorption of heavy metals in aqueous solution. Water Research, 38(11), 2643-2650. http://dx.doi.org/10.1016/j. watres.2004.03.026. PMid:15207594.

36. Brasil. Ministério do Meio Ambiente. Conselho Nacional do Meio Ambiente. (2011, 13 de maio). Resolução CONAMA $n^{\circ} 430$, de 13 de maio de 2011. Diário Oficial da República Federativa do Brasil, Brasília.

37. Brasil. Conselho Nacional do Meio Ambiente. (2005, 17 de março). Resolução CONAMA n ${ }^{\circ} 357$, de 17 de março de 2005. Diário Oficial da República Federativa do Brasil, Brasília.

38. Robinson-Lora, M. A., \& Brennan, R. A. (2011). Anaerobic precipitation of manganese and co-existing metals in mine impacted water treated with crab shell-associated minerals. Applied Geochemistry, 26(5), 853-862. http://dx.doi.org/10.1016/j. apgeochem.2011.02.006.

Enviado: Abr. 22, 2014

Revisado: Set. 22, 2014

Aceito: Jan. 22, 2015 